\title{
Cytochrome c oxidase deficiency, oxidative stress, possible antioxidant therapy and link to nuclear DNA damage
}

\author{
Liza Douiev ${ }^{1,2} \cdot$ Bassam Abu-Libdeh ${ }^{3} \cdot$ Ann Saada ${ }^{1,2}$
}

Received: 18 October 2017 / Accepted: 27 October 2017 / Published online: 2 February 2018

(c) European Society of Human Genetics 2018

\begin{abstract}
In response to Ravera et al. "Fanconi anemia: from DNA repair to metabolism" commenting on our recent publication by Abu-Libdeh, Douiev et al., describing a pathogenic variant in the $C O X 4 I 1$ gene simulating Fanconi anemia, we wish to add supplementary, pertinent information linking cytochrome $c$ oxidase (COX, mitochondrial respiratory chain complex IV) dysfunction to oxidative stress and nuclear DNA damage. Elevated production of reactive oxygen species (ROS) in $C O X 4 I 1$ deficient fibroblasts was detected in cells grown in glucose free medium and normalized by ascorbate or N-acetylcysteine supplementation. A pilot study shows positive nuclear staining with antibodies against Phospho-Histone H2A.X (Ser 139) indicating double-stranded DNA breaks (DBSs) both in COX $4 I 1$ and in COX6B1 deficient fibroblasts. Additional investigation is required, and ongoing, to elucidate the precise mechanism of DNA damage in mitochondrial respiratory chain dysfunction and how it could be prevented.
\end{abstract}

\section{Introduction}

We appreciate the comments of Ravera et al. "Fanconi anemia: from DNA repair to metabolism" [1] to our recent publication by Abu-Libdeh, Douiev et al., "Mutation in COX4I1 gene, is associated with short stature, poor weight gain and increased chromosomal breaks, simulating Fanconi Anemia." describing a novel form of cytochrome $c$ oxidase (COX, mitochondrial respiratory chain complex IV) defect simulating Fanconi anemia (FA) due to a pathogenic variant in the $C O X 4 I 1$ gene [2]. COX4II encodes the common isoform of COX4 subunit which has a regulatory function in $\mathrm{COX}$ that catalyzes the final electron transfer step in the mitochondrial respiratory chain, the major oxygen consumer. Thus, impaired COX could

$\triangle$ Ann Saada

annsr@hadassah.org.il

anns@ekmd.huji.ac.il

1 Monique and Jacques Roboh Department of Genetic Research, Hadassah-Hebrew University Medical Center, Jerusalem, Israel

2 Department of Genetic and Metabolic Diseases, Hadassah-Hebrew University Medical Center, Jerusalem, Israel

3 Department of Pediatrics, Makassed Hospital and Al-Quds University, Palestinian Authority, Jerusalem, Israel potentially lead to the accumulation of reactive oxygen species (ROS) and oxidative stress [3]. Accordingly, Ravera and colleagues suggest to shift the focus in FA, from defects from DNA repair to energy metabolism and suggest that heterogeneity of the disease fits with a complex metabolic defect rather than with a mere DNA-repair disease [1]. They also highlight the possibility of antioxidant treatment in FA. Also, we and others have noted the link between principal FA mutations in genes linked to DNA repair, mitochondrial dysfunction, and oxidative stress. Reciprocally, identification of the COX $4 I 1$ defect demonstrated that a primary respiratory chain defect can induce the hallmark sign of FA, a positive chromosomal fragility test (discussed in refs. [1, 2]). Notably, bone marrow failure is also a feature of some other COX deficiencies [4] but it is presently unclear if DNA damage is present in these other respiratory chain defects. In this context, we present the results of a pilot study, aimed to investigate oxidative stress, the effects of antioxidants, and signs of nuclear DNA damage in COXdeficient cells.

\section{Materials and methods}

Previously established skin fibroblasts cell lines [2, 5] were maintained in permissive high-glucose DMEM supplemented with fetal calf serum (FCS) pyruvate and uridine 
(GLU) [4]. To evaluate ROS production, by $\mathrm{H}_{2}$ DCFDA (DCF), cells were cultivated in microtiter wells in GLU and in glucose-free DMEM medium containing dialyzed FCS and galactose (GAL) for $72 \mathrm{~h}$ in the absence or presence of either $12 \mu \mathrm{M}$ Ascorbate, $1 \mathrm{mM}$ N-acetylcysteine (NAC), or $0.5 \mathrm{mM}$ 5-aminoimidazole-4-carboxamide ribonucleotide (AICAR) and normalized to cell content measured by methylene blue, as we have previously described [6]. Measurements were done in triplicates on at least two different occasions and results were analyzed by 2-tailed Student's $t$-test.

Double-stranded DNA breaks (DBSs) were evaluated by staining cells grown on coverslips with antibodies against Phospho-Histone H2A.X (Ser 139) using the Oxiselect DNA Double stranded Break (DBS) Staining Kit according to the manufacturer's instructions (Cell Biolabs Inc., Sand Diego, CA, USA). Positive control was treated with $100 \mu \mathrm{M}$ Etopoiside for $1 \mathrm{~h}$ prior to staining. Nuclei were stained with Hoechst 33342, NucBlue live cell stain (Molecular probes, Life technologies Eugene OR USA). Preparates were mounted with Fluoromunt-G (SouthernBiotech, Birmingham AL, USA) and examined by confocal microscopy (Nikon A1R). The percentage of nuclei with DBSs was calculated by observing over a hundred nuclei.

\section{Results}

We have previously reported preliminary data, observing elevated ROS production in $C O X 4 I 1$ deficient fibroblasts grown in glucose-free GAL where energy production is dependent on the mitochondrial respiratory chain, whereas in GLU medium, ROS production did not differ from normal control cells [2]. We have now repeated and quantified the data which are depicted in Fig. 1a. Growth on GAL medium was also significantly affected as seen by the decreased GAL:GLU growth ratio, which is consistent with mitochondrial respiratory chain dysfunction (Fig. 1b). Mitochondrial membrane potential was not significantly affected (results not shown) in either medium. The effects of two antioxidant molecules were examined and both ascorbate and NAC normalized the ROS production on GAL. Only NAC had a significant positive effect on the growth ratio. AICAR did not affect ROS production or growth. Staining with anti-Phospho-Histone H2A.X (Ser 139) revealed markedly elevated occurrence of DBSs in the nuclei both in $C O X 4 I 1$ (73\% positive stain) and COX6B1 (96\% positive stain) deficient fibroblasts compared to normal control cells (24\%). Notably, these initial results were obtained with cells grown on GLU, and ongoing studies will reveal the extent of DBSs in GAL medium and the effect of antioxidants (Fig. 2).

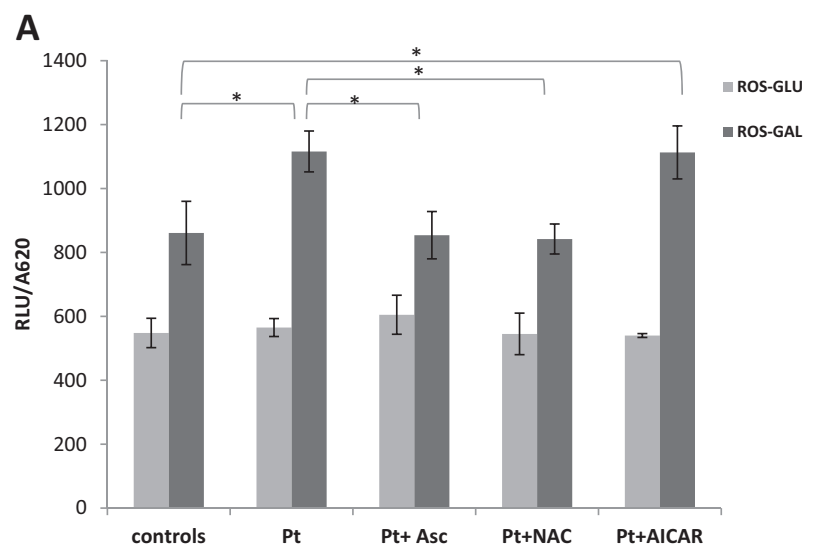

B

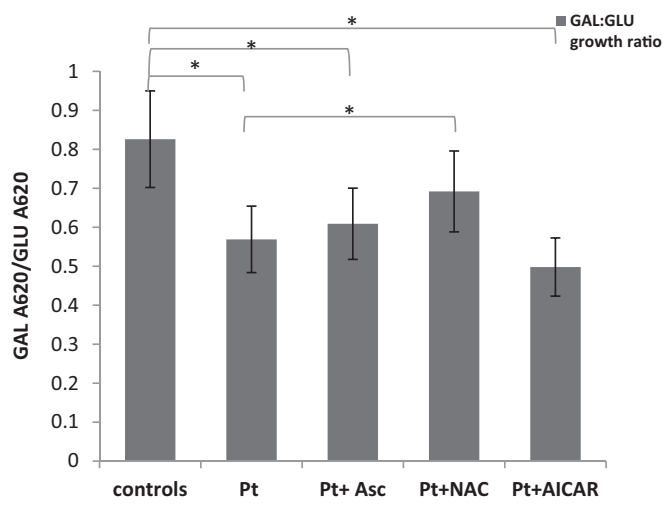

Fig. 1 ROS production and growth ratio. COX $4 I 1$ deficient fibroblast (Pt) were grown in GLU or GAL medium in the absence or presence of Ascorbate $(\mathrm{Pt}+\mathrm{Asc})$, NAC $(\mathrm{Pt}+\mathrm{NAC})$, or AICAR $(\mathrm{Pt}+\mathrm{AICAR})$ for $72 \mathrm{~h}$. a ROS production was measured and normalized to cell content (RFU/A620). b Ratios of cell content on GAL:GLU (GAL A620/GLU A620) were calculated. The results are presented as mean \pm SEM; $* p<0.05$

\section{Discussion}

Our results in $C O X 4 I 1$ deficient cells show that although oxidative stress might initially not be evident, it can be induced under stressful conditions and demonstrate the possibility of antioxidants treatment to lessen excess ROS production. We have previously noted the positive effect of Ascorbate and NAC in another more severe form of COX deficiency, due to mutated COX6B1 and a number of other respiratory chain deficiencies [5-7]. Notably, Ascorbate and NAC were chosen for the present experiments as they are readily available and safe to use. AICAR, which is not an antioxidant, but was reported to be beneficial in both COX and other mitochondrial respiratory chain deficiencies due to activation of the AMPK/PGC- $1 \alpha$ axis $[6,8]$ did not have any significant effect. Thus, on the basis of the above data, the COX 4I1 patient has now initiated Ascorbate (vitamin C) supplementation, and her status will be evaluated in the coming months. Additionally, our result shows that nuclear DNA damage is not only confined to blood cells but is also 
Merge
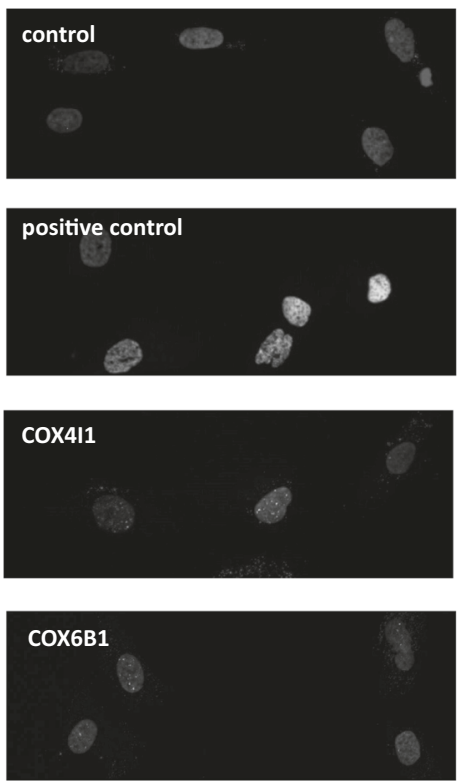

Fig. 2 Double-stranded DNA breaks. Representative micrographs $\times 60$ magnification, of control, $C O X 4 I 1$, and COX6B1 fibroblasts grown overnight, stained with anti-Phospho-Histone H2A.X (Ser 139)

evident in the fibroblasts. Notably, the occurrence of DBSs was higher in COX6Blcells where COX activity was severely decreased [5], and less in COX $4 I 1$ cells where COX activity was only partially affected [2]. Our results are also in accord with Liang et al. linking mitochondrial damage with decreased COX activity to impaired DNA break repair [9]. Ongoing and future investigation will reveal if DBSs are present also in other respiratory chain deficiencies, under what conditions and how they can be prevented/lessened.

Acknowledgements A.S. is supported by the Pakula family via AFHU. Dr. Yael Feinstein-Rotkopf from the Core Research Facility, Hebrew University Faculty of Medicine, is acknowledged for expert assistance with confocal microscopy.

\section{Compliance with ethical standards}

Conflict of interest The authors declare that they have no conflict of interest.

\section{References}

1. Ravera S, Dufour C, Degan P, Cappelli E. Fanconi anemia: from DNA repair to metabolism [letter]. Eur J Hum Genet. 2018, this issue.
anti-Phospho-Histone H2AX(Ser 139)
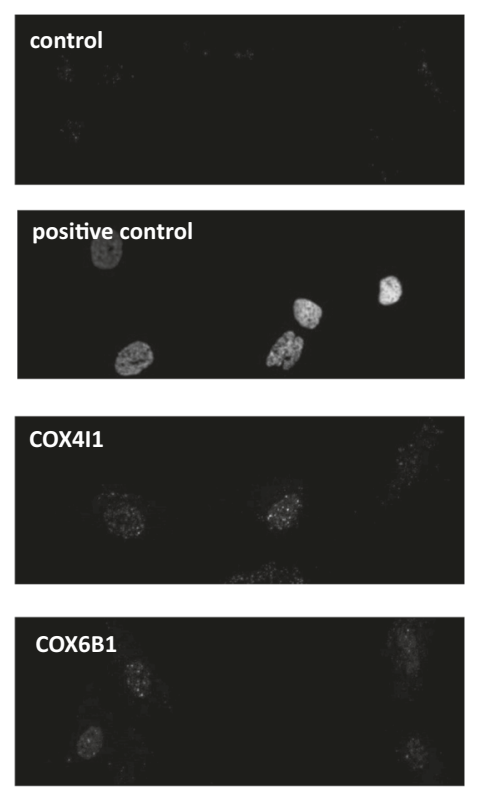

antibodies, and with Hoechst 33342. Positive control were normal fibroblasts treated with Etopoiside prior to staining

2. Abu-Libdeh B, Douiev L, Amro S, Shahrour M, Ta-Shma A, Miller C, et al. Mutation in the COX4I1 gene is associated with short stature, poor weight gain and increased chromosomal breaks, simulating Fanconi anemia. Eur J Hum Genet. 2017;25:1142-46.

3. Bourens M, Fontanesi F, Soto IC, Liu J, Barrientos A. Redox and reactive oxygen species regulation of mitochondrial cytochrome C oxidase biogenesis. Antioxid Redox Signal. 2013;19:1940-52.

4. Rak M, Bénit P, Chrétien D, Bouchereau J, Schiff M, El-Khoury R, et al. Mitochondrial cytochrome c oxidase deficiency. Clin Sci (Lond). 2016;130:393-7.

5. Abdulhag UN, Soiferman D, Schueler-Furman O, et al. Mitochondrial complex IV deficiency, caused by mutated COX6B1, is associated with encephalomyopathy, hydrocephalus and cardiomyopathy. Eur J Hum Genet. 2015;23:159-64.

6. Golubitzky A, Dan P, Weissman S, Link G, Wikstrom JD, Saada A. Screening for active small molecules in mitochondrial complex I deficient patient's fibroblasts, reveals AICAR as the most beneficial compound. PLoS ONE. 2011;6:e26883.

7. Douiev L, Soiferman D, Alban C, Saada A. The effects of ascorbate, $\mathrm{N}$-acetylcysteine, and resveratrol on fibroblasts from patients with mitochondrial disorders. J Clin Med. 2016;6:pii: E1 https:// doi.org/10.3390/jcm6010001.

8. Viscomi C, Bottani E, Civiletto G, Cerutti R, Moggio M, Fagiolari $\mathrm{G}$, et al. In vivo correction of COX deficiency by activation of the AMPK/PGC-1 $\alpha$ axis. Cell Metab. 2011;14:80-90.

9. Liang DH, Choi DS, Ensor JE, Kaipparettu BA, Bass BL, Chang JC. The autophagy inhibitor chloroquine targets cancer stem cells in triple negative breast cancer by inducing mitochondrial damage and impairing DNA break repair. Cancer Lett. 2016;376:249-58. 